\title{
In-situ Online Measurement of Rhombic Distortion in Billets
}

\author{
Prabal Patra, Ashish Tiwari, Punit Rathore \\ Instrumentation \& Control, Automation Division, Tata Steel Jamshedpur, India
}

Email address

prabal.patra@tatasteel.com (P. Patra)

\section{To cite this article:}

Prabal Patra, Ashish Tiwari, Punit Rathore. In-situ Online Measurement of Rhombic Distortion in Billets. Engineering Science. Vol. 5, No. 2, 2020, pp. 10-23. doi: 10.11648/j.es.20200502.11

Received: July 5, 2019; Accepted: July 26, 2019; Published: May 28, 2020

\begin{abstract}
DD (diagonal-difference) is considered as measure of rhombic distortion, aka Rhomboidity, which is a shape related defect in square cross-section billets. Rhomboidity in billets starts with non-uniform shell solidification in the mold primarily due to inconsistent cooling causing irregular heat transfer. The higher diagonal difference greatly impacts the quality of billets to be rolled at various mills. Rhomboidity at or over $4 \%$ leads to billet twisting in the roughing stands of the rolling mill. Currently, billet rhomboidity is measured manually at end of casting operation. The presented work describes an optical, online \& real-time image processing based method to determine the rhomboidity induced in each strand and alerts the operator to take corrective actions. The online Rhomboidity Measurement System employs sophisticated image acquisition \& processing techniques to determine face contours of the billet with sub-pixel accuracy. The key features of RMS are the construction of a gaussian penalty function for selection of suitable 4-lines combination that precisely fits the billet face and use of a highly efficient and accurate statistical indicator, based on KL-Divergence measure, to estimate the rhomboidity even in presence of partial occlusion of billet face by scales. The expected savings are to the tune of 0.27 Million USD.
\end{abstract}

Keywords: Rhombic Distortion in Billets, Shape Defects in Continuous Casting, Quadrilateral Detection, Hough Transform, Gaussian Penalty Function, KL-divergence Measure

\section{Introduction}

Steel is made in steel melting shop in the refractory lined vessels called LD Converters by blowing oxygen through the hot metal bath. While iron making is a reduction process, steel making is an oxidation process. Within the continuous casting sub-process of the steel manufacturing process, where the molten steel is passed through a water-cooled near-vertically-aligned lubricated mold of rectangular cross-section about a meter long to emerge in the form of a continuous strand consisting of a solidified shell encapsulating molten material, this strand being further cooled to complete solidification using water sprays even as the orientation is changed to horizontal using rollers $[1,2]$.

Billet Rhomboidity starts with non-uniform shell solidification in the mold which is due to inconsistent mold cooling causing irregular heat transfer, therefore inducing thermal stress, making the emerging out billet face to be skewed to a have rhombus cross-sectional shape [2]. The higher diagonal difference greatly impacts the quality of billets to be rolled at various mills. Rhomboidity at or over $4 \%$ leads to billet twisting in the roughing stands of the rolling mill.

Generation of rhombus shape billets adversely affects the productivity by creating mill stoppages, increases the energy consumption due to reheating requirement of colder billets in downstream processing, increases the yield loss on account of rejection of huge volumes of input material by customer and creates an unsafe environment due to extensive material handling requirements for manual inspection.

In absence of any reliable and proven alternative, conventionally, worldwide, the billets are measured manually on random sample basis every four hours. It's a delayed feedback process as it takes at-least 4 hours for the billet to cool down to a suitable temperature where the measurement can be taken, and only after that, necessary corrective action can be initiated. Once the corrective action is taken, it takes another 4 hours to get the confirmation of the effect. In this process, batch of heats of 8 hours (approx. 1200 tons or 960 billets) are kept on hold. Handling, sorting and inspection of batch of almost 1000 billets become a huge task. Hence, to reduce rejection due to Rhomboidity defect and unsafe condition thus generated in handling billets, an online measurement system is required that will reduce time of inspection, batch holds and help immediate corrective actions to be taken. 
Patent US4538669A, "Distortion measurement in casting", describes a contact type mechanism to determine the Rhomboidity. In the invention, the authors Kegham M. Markarian \& Robert Sobolewski [3] propose to use pair of contact rollers supported by respective guide arms. The feedback about the respective positions and the separation between the rollers, Figure 1, is used to determine the Rhomboidity index. However, this device is highly maintenance prone due to its contact-type nature with high temperature billet and prone to mechanical damages. Also, the accuracy diminishes with time due to wear \& tear of couplers, springs and other mechanical components.

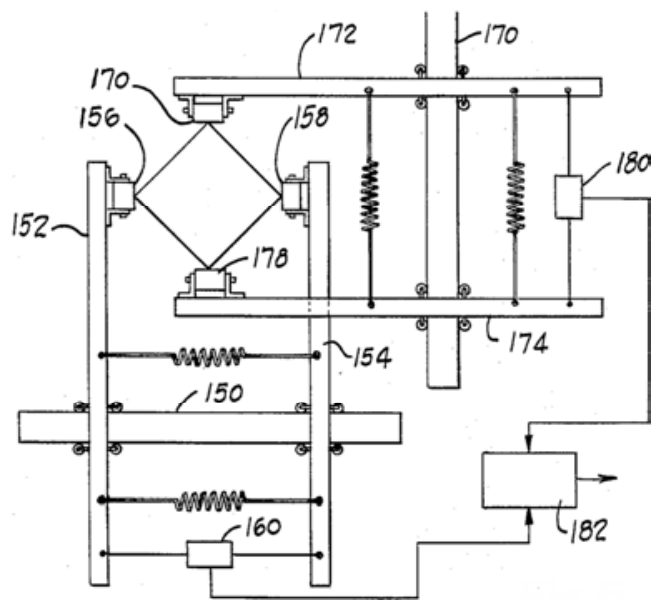

Figure 1. Schematic description of working of contact type roller for rhombic distortion measurement.

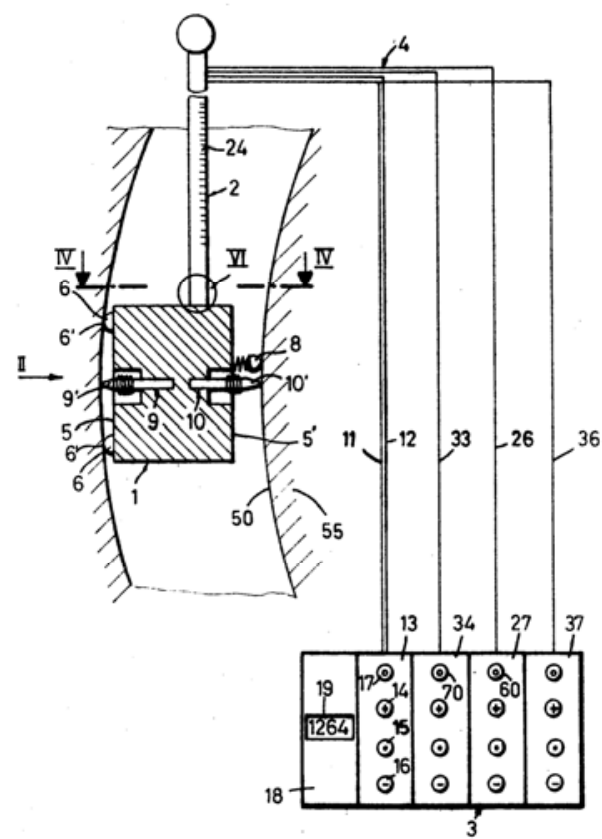

Figure 2. Mold geometry measurement apparatus and description of principle of operation.

A related technique was proposed by Marcus Schmid \& Adolf Fuchs [4], US4087918A, wherein the proposed apparatus for measuring the geometry of the hollow mold compartment of continuous casting molds comprised of a measuring head displaceable essentially in the direction of its lengthwise axis and having three impacts or contact elements, as shown in Figure 2, arranged at a first side of the measuring head.

The impact elements form a triangle which is parallel to the lengthwise axis of the measuring head. A first measuring feeler is arranged at this first side so as to bear against the neighboring mold wall and is perpendicular to the plane of the triangle. A second measuring feeler is arranged at the second side of the measuring head coaxial to the first measuring feeler and bears against the opposite wall of the hollow mold compartment. Indicator elements operatively connected with the measuring feelers indicate the position thereof. However, this method is totally offline and it can only measure the mold geometry once, before the start of operation, and is incapable of doing any measurements when the caster is in operation. Moreover, in case of any fault, repair activity must wait till the end of the ongoing production schedule.

Rhomboidity, therefore, is a serious problem for steel manufactures and needs to be controlled anyhow as the current method of manual inspection and other existing techniques are not very effective and generate problems of its own. A method is needed to measure the rhombic distortion in billets at the time of casting itself. So that immediate corrective actions can be taken and further defect generation can be avoided. Such a method should be accurate, real-time, non-contact type, less maintenance prone and must be equipped with alarms to indicate rhomboidity almost immediately. The organization of our paper is as follows: Relevant theories and mathematical preliminaries are given in Section II, Automated Rhomboidity measurement system is described in Section III, Algorithm and experimental results are shown in Section IV, Section V discusses the benefits achieved thereof and conclusion and way forward is presented in Section VI.

\section{Relevant Theory \& Mathematical Prelimineries}

This section describes the basic theories and mathematical preliminaries used in building the image processing algorithm for detection of billet face contours with sub-pixel accuracy.

A. Hough transform for line detection in images

The Hough transform was originally developed to recognize lines [5] and has later been generalized to cover arbitrary shapes $[6,7]$. The advantage of the Hough transform is that the pixels lying on one line need not be contiguous. This can be very useful when trying to detect lines with short breaks in them due to noise, or when objects are partially occluded. This is extremely important in our case as the emergent billet temperature is around $900{ }^{\circ} \mathrm{C}$ and, at this temperature, it reacts with ambient oxygen to form flakes of iron oxides which further get deposited on the surface as black patches. Presence of flakes make the Image processing of billet face extremely difficult as it partially occludes the edges.

A. 1. Representation of Lines in the Hough Space

Lines can be represented uniquely by two parameters. Often the form in Equation (1) is used with parameters a and b [5]. 


$$
y=a \cdot x+b
$$

This form is, however, not able to represent vertical lines. Therefore, the Hough transform uses the form in Equation (2), which can be rewritten to Equation (3) to be Equation (1). The parameters $\theta$ and $r$ is the angle of the line and the distance from the line to the origin respectively.

$$
\begin{gathered}
r=x \cdot \cos \theta+y \cdot \sin \theta \Leftrightarrow \\
y=-\frac{\cos \theta}{\sin \theta} \cdot x+\frac{r}{\sin \theta}
\end{gathered}
$$

All lines can be represented in this form when $\theta \in$ $[0,180]$ and $r \in R($ or $\theta \in[0,360]$ and $r \geq 0)$. The Hough space for lines has therefore these two dimensions; $\theta$ and $r$, and a line is represented by a single point, corresponding to a unique set of parameters $\left(\theta_{0}, r_{0}\right)$. The line-to-point mapping is illustrated in Figure 3.

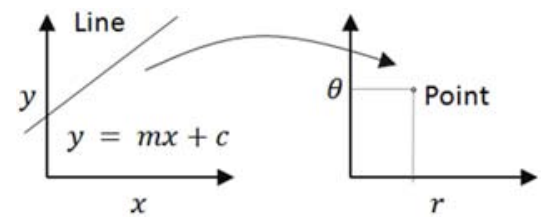

Figure 3. A line in cartesian plane is represented as a point in Hough Space or $(r, \theta)$ space.

\section{A. 2. Algorithm for line detection in image}

The algorithm for detecting straight lines can be divided into the following steps:

1. Edge detection, e.g. using the Canny edge detector [8].

2. Mapping of edge points to the Hough space and storage in an accumulator.

3. Interpretation of the accumulator to yield lines of infinite length. The interpretation is done by thresholding and possibly other constraints.

4. Conversion of infinite lines to finite lines.

Typical Hough accumulator matrix [7] is shown in Figure 4. This distribution can be multimodal depending on number of lines present in the image.

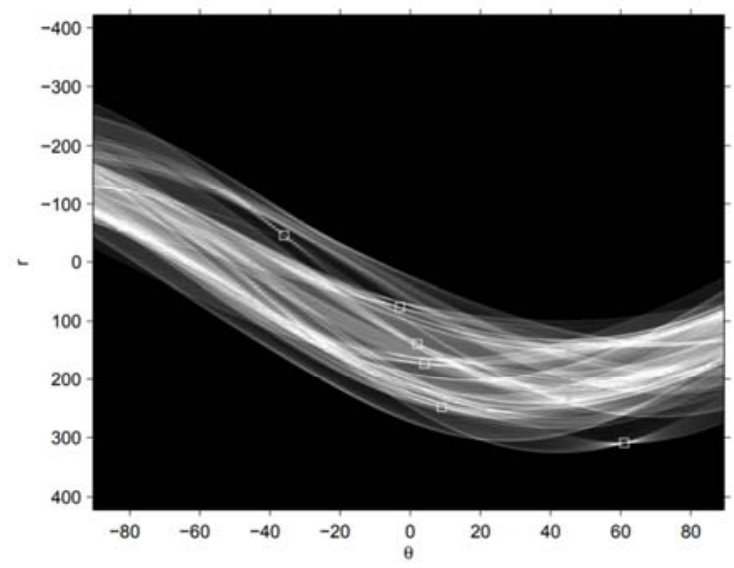

Figure 4. Image representation of Hough accumulator matrix. The peaks are shown as square blocks corresponding to lines in the image.

The finite lines can then be superimposed back on the

original image. The Hough transform itself is performed in point 2 , but it further requires finding peaks, corresponding to the lines in the image, in Hough accumulator matrix. While searching for 'true' peaks in that space several constraints are used to remove detection of very short lines, noise etc.

B. Kullback-Leibler divergence measure

The $K L$ divergence [9], which is closely related to relative entropy, information divergence, and information for discrimination, is a non-symmetric measure of the difference between two probability distributions $p(x)$ and $q(x)$. Specifically, the Kullback-Leibler $(K L)$ divergence of $q(x)$ from $p(x)$, denoted $D_{K L}(p(x), q(x))$, is a measure of the information lost when $q(x)$ is used to approximate $p(x)$.

Let $p(x)$ and $q(x)$ are two probability distributions of a discrete random variable $x$. That is, both $p(x)$ and $q(x)$ sum up to 1 , and $p(x)>0$ and $q(x)>0$ for any $x$ in $X$. $D_{K L}(p(x), q(x))$ is defined in Equation (4).

$$
D_{K L}(p(x), q(x))=\sum_{x} p(x) \ln \frac{p(x)}{q(x)}
$$

Typically, $p(x)$ represents the "true" distribution of data, observations, or a precisely calculated theoretical distribution. The measure $q(x)$ typically represents a theory, model, description, or approximation of $p(x)$.

Although the $K L$ divergence measures the "distance" between two distributions, it is not a distance measure [10]. This is because that the $K L$ divergence is not a metric. It is not symmetric: the $K L$ from $p(x)$ to $q(x)$ is generally not the same as the $K L$ from $q(x)$ to $p(x)$. Furthermore, it need not satisfy triangular inequality. Nevertheless, $D_{K L}(P \| Q)$ is a non-negative measure. $D_{K L}(P \| Q) \geq 0$ and $D_{K L}(P \| Q)=0$ if and only if $P=Q$.

This is used for computing 'Rhombic distortion index' as the $K L$-divergence between the probability mass function estimated from observed values from a given strand and the probability mass function of sample values where it was known that caster was producing normal shape billets.

\section{System Description}

Essentially the work presented here is design and development of a Machine Vision System comprising of a high resolution, high speed industrial matrix CCD scanner, installed inside a double layered stainless steel enclosure with adequate water cooling and air purging facility, a data acquisition card to capture billet/strand identification signals and billet resting signal to initiate image acquisition and processing thereof and a computing unit (workstation) which hosts image processing software to analyze the image captured and determine the $\%$ diagonal difference of the billet face captured in the image.

(a)

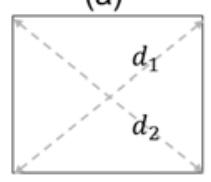

Figure 5. (a) Prefect billet shape, $d_{1}=d_{2}$. (b) Rhombus billet shape $d_{1}>d_{2}$. (b)

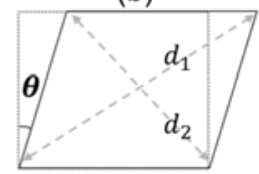


Conventionally, Rhomboidity is defined as below:

$$
R=\frac{\left(d_{1}-d_{2}\right)}{\left(d_{1}+d_{2}\right)} \times 100
$$

Where $d_{1}$ and $d_{2}$ are the length of the opposite diagonals of a rhombus. Rhomboidity at or over $4 \%$ leads to twisting of billets in downstream processing.

The camera, enclosure and lens (image acquisition system) is installed adjacent to the first V-shaped notch of the transfer bed. It captures the face of each billet as it moves on. High precision machined camera mounting plate ensures that the CCD plane of the camera is exactly parallel to the face of the billet to avoid any perspective distortion errors. A combination of PLC signals, viz., LIFT, HOLD, PUSH and MOVE are programmed in a manner to associate the billet face being observed by the camera at any instance of time with its strand number. Using this innovative identification mechanism, the billets of all 6 strands of the caster can be sequentially measured for rhombic distortions using a single system only, which would have otherwise required 6 different installations.

Protecting the Optoelectronic devices from heat irradiant from freshly cast hot billets was a challenge in its own. The entire thing is enclosed in doubly layered stainless-steel chamber which has inlet/outlet provision for cold water. This ensures the electronics is protected inside and the residual heat is taken out by blowing clean instrument air. This emergent air from the enclosure creates a high-pressure zone around enclosure front lens and minimizes the amount of dust accumulated thereof.

Once billet rest signal is received, system captures the image of the billet face and further using Hough transform determines all the possible lines in image, say N. Exhaustive search is performed on all possible combination of 4 lines, ${ }^{N} C_{4}$, to find its suitability for fitting an (almost) quadrilateral face. Gradual penalty function based on Gaussian distribution has been devised for this purpose and found to work extremely well under the given conditions. The penalty scores are based on various parameters determined by known geometry of the billets. For example, distance between parallel lines, angle between perpendicular lines, total area enclosed inside the 4-line combination etc. Once the fitting contour is obtained, it is further fine-tuned to define the true billet face boundaries and from there all the measurements including DD are obtained.

Due to high temperature of the freshly cast billet, around $900^{\circ} \mathrm{C}$, the outer surface of the billet oxidizes fast and gets deposited as flakes on the surface, commonly known as scales. Presence of scales occludes the face of the billet partially and therefore makes the contour detection extremely difficult and measurements thus obtained would not be useful for any decision-making purposes.

One possible solution is to use especially designed air nozzles to blow off scales deposited on billet face before the it reaches in the field of view of the camera for measurements. Although this can solve the problem, it reduces the ability of the system to determine active face contour of the billet faithfully due to increased cooling rate of billets due to air purging.
The solution that finally mitigated this issue came in form of a statistical method where histogram shape of DD measurements of individual strands was taken into account and the abnormality index was calculated based on the shape difference of histogram of a strand with respect to shape of an ideal histogram. The ideal histogram shape is obtained by having measurements of the billets which are known to be free from any rhombic distortions. The KL-divergence method has been used for calculating the shape difference between histograms. This method induces 20 minutes delay in the decision process but is more than acceptable for all operational purposes.

\section{Algorithm \& Results}

The stepwise procedure adopted to determine billet rhombic index is as described below.

\section{Image Acquisition \& Billet Face Localization}

The crux of the innovation lies in the design of imaging setup and in building image processing algorithms to faithfully detect the billet face corners. The red-hot billet (approx. at $900^{\circ} \mathrm{C}$ ) emits radiation in near infrared and visible region [11]. The relation between spectral radiation and the temperature of an object is governed by Plank's equation as given below in Equation (6):

$$
u(\lambda, T)=\frac{8 \pi h c}{\lambda^{5}} \frac{1}{e^{\frac{h c}{\lambda k T}-1}}
$$

Where, $\quad c=2.997924 \times 10^{8} \mathrm{~m} / \mathrm{s}, \quad k=1.38064 \times$ $10^{-23} \mathrm{~J} / K$, and $h=6.626069 \times 10^{-34} \mathrm{~J} . \mathrm{s}$

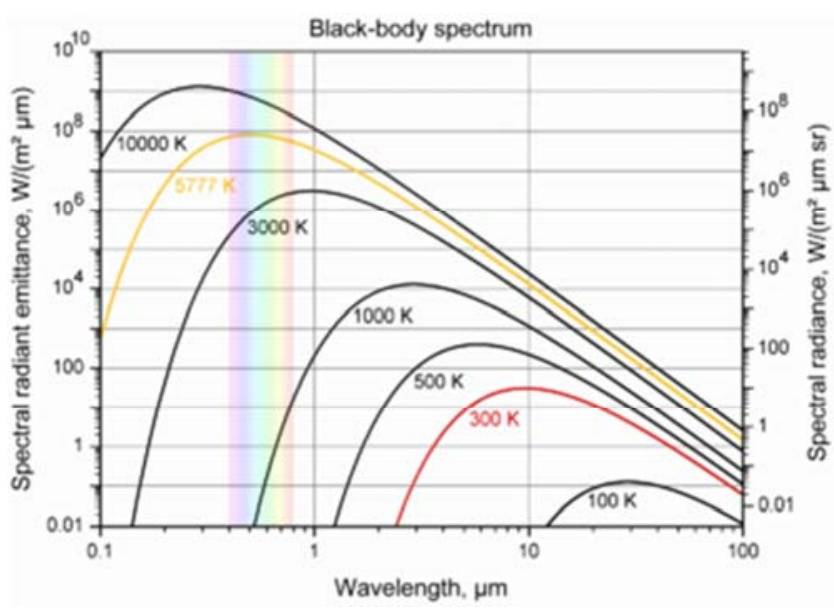

Image source: https://sv.wikipedia.org/wiki/Svartkropp.

Figure 6. Characteristic spectral emission curve for objects (black body) at different temperatures.

The IR radiation, only due to temperature, from red-hot billet sufficiently excites the CCD cells to accumulate charge but contributes nothing towards the shape information about the object to be measured. This is clearly a problem. If we closely observe, all the surface texture information, including edges, of a red-hot object, independent of its material, is washed out. In-fact, the edges of billet face would appear swollen that would make line detection even more tedious. To overcome this problem, the imaging system uses an IR cut filter, $650 \mathrm{~nm}$, and 
a high-power illumination source $(\lambda \in(480,550) \mathrm{nm})$ to swamp out the undesirable IR radiation. Figure 6 shows the relationship between the object temperature and the characteristic spectral radiation as observed from the object:
Billet face localization is straight-forward as it can only move slightly from its rest position on the V-shaped notch. Segmented weighted averaging of pixel blocks accurately localizes the billet face in the acquired image.

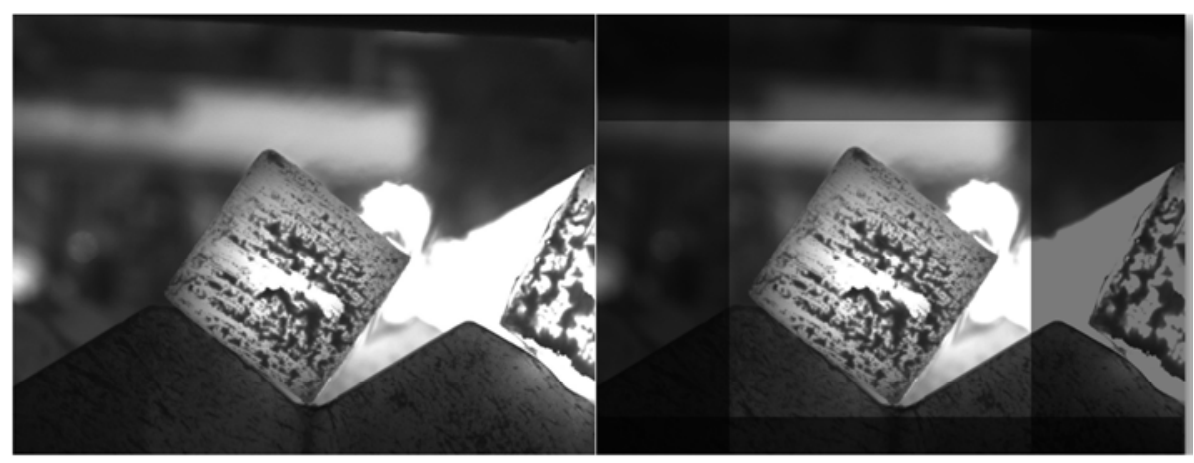

(a)

(b)

Figure 7. Billet image processing. (a) Original image. (b) billet face localization.

2. Edge Map \& Lines Detection

Variation in billet temperature \& the variation in ambient illumination level throughout the day, together, give rise to variations in the quality of images being captured.

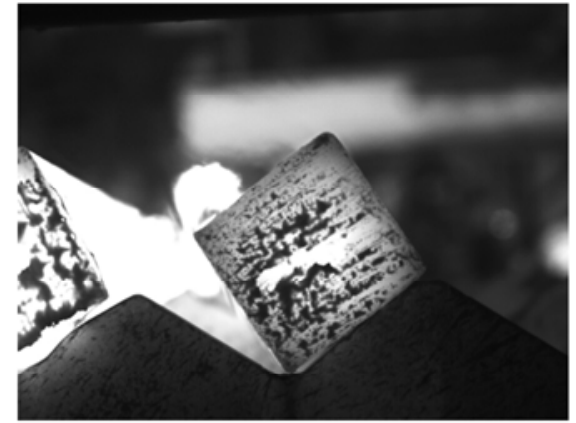

(a)

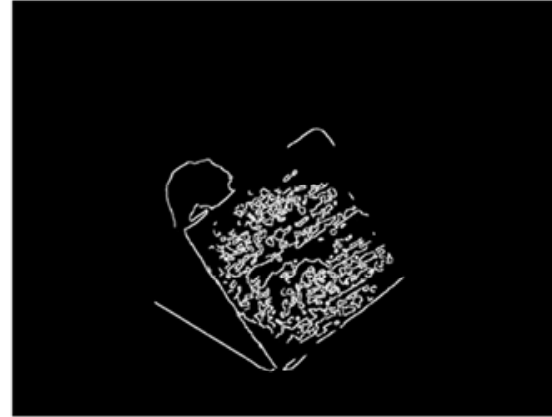

(b)

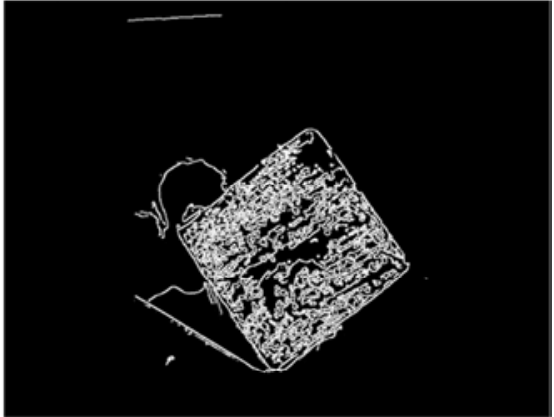

(c)

Figure 8. Adaptive edge detection. (a) Original image. (b) normal edge detection. (c) adaptive edge detection.

Adaptive edge detection is needed to account for the variance in the brightness levels and hence the gradient values inside the image. We have used canny edge detector with varying threshold levels based on the illumination conditions to ensure reliable edge detection. The results of adaptive edge detection is as shown in Figure 8.

Hough transform has been used to find lines in the image. It works on the edge map obtained by the adaptive canny edge detector. For noisy data, Hough transform is statistically superior than least square line fitting.

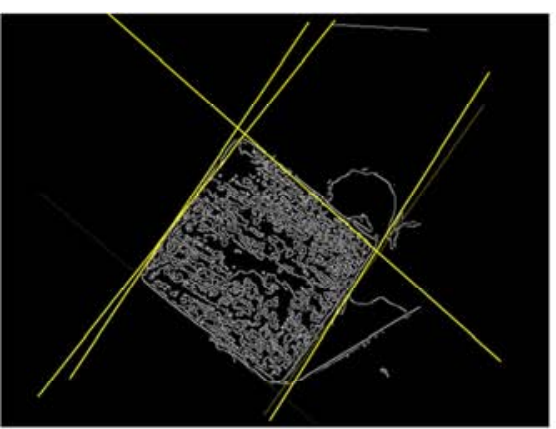

(a)

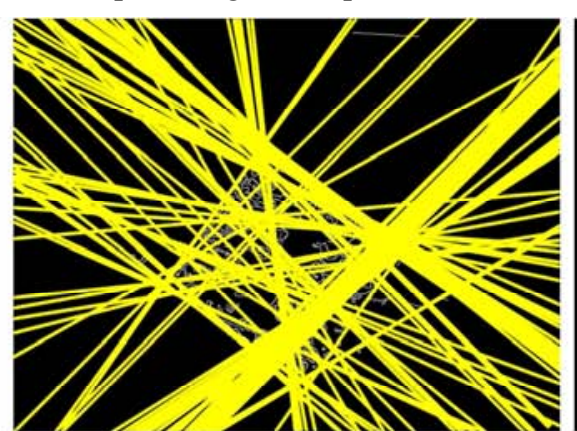

(b)

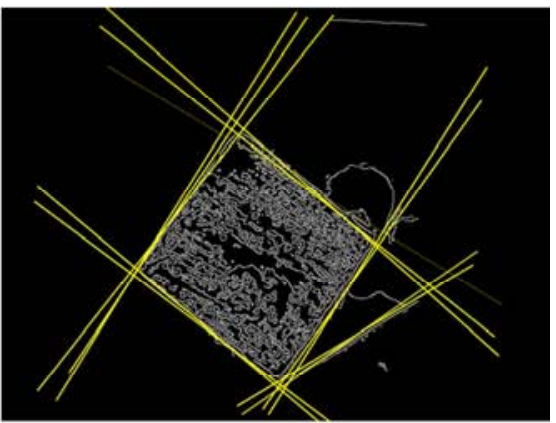

(c)

Figure 9. Detection of lines in the image using Hough transform. Relaxation in criteria increases number of lines to be detected.

Lines in the image have been detected by searching for peaks in Hough accumulator matrix. To clean the data, all the smaller peaks, corresponding to less than 20 contributing edge points, were omitted from the result. Further, out of total number of detected lines, $\mathrm{N}$, a set $\mathbb{V}$ was constructed that consists of all possible 4-line combinations, which in later step, 
evaluated for its suitability for fitting the billet face. Here it is important to mention the computational complexity that is involved in searching the space for optimum 4-line solution.

For example, let total no of detected lines were $(\mathrm{N})=100$;

Then,

$$
|\mathbb{V}|={ }^{100} C_{4}=3921225
$$

3. Best Fit Quadrilateral Search

Here, we now introduce our Gaussian penalty function for giving scores to each 4-line combination according to their appropriateness for fitting the face contours of billet. From, each 4-line combination the following parameters are extracted,

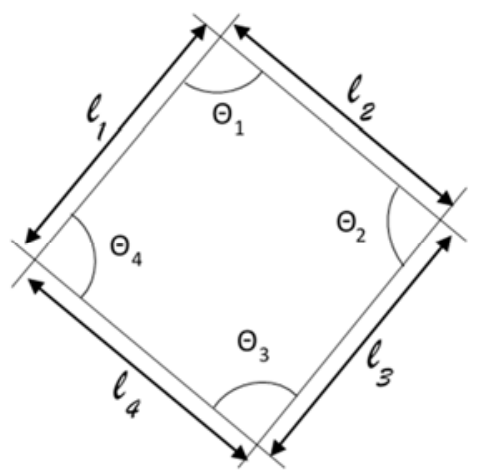

Figure 10. Every 4-line combination yields 9 element vector.

$$
x=\left(l_{1}, \theta_{1}, l_{2}, \theta_{2}, l_{3}, \theta_{3}, l_{4}, \theta_{4}, A\right)
$$

Where, $A$ represents the area enclosed inside the point of
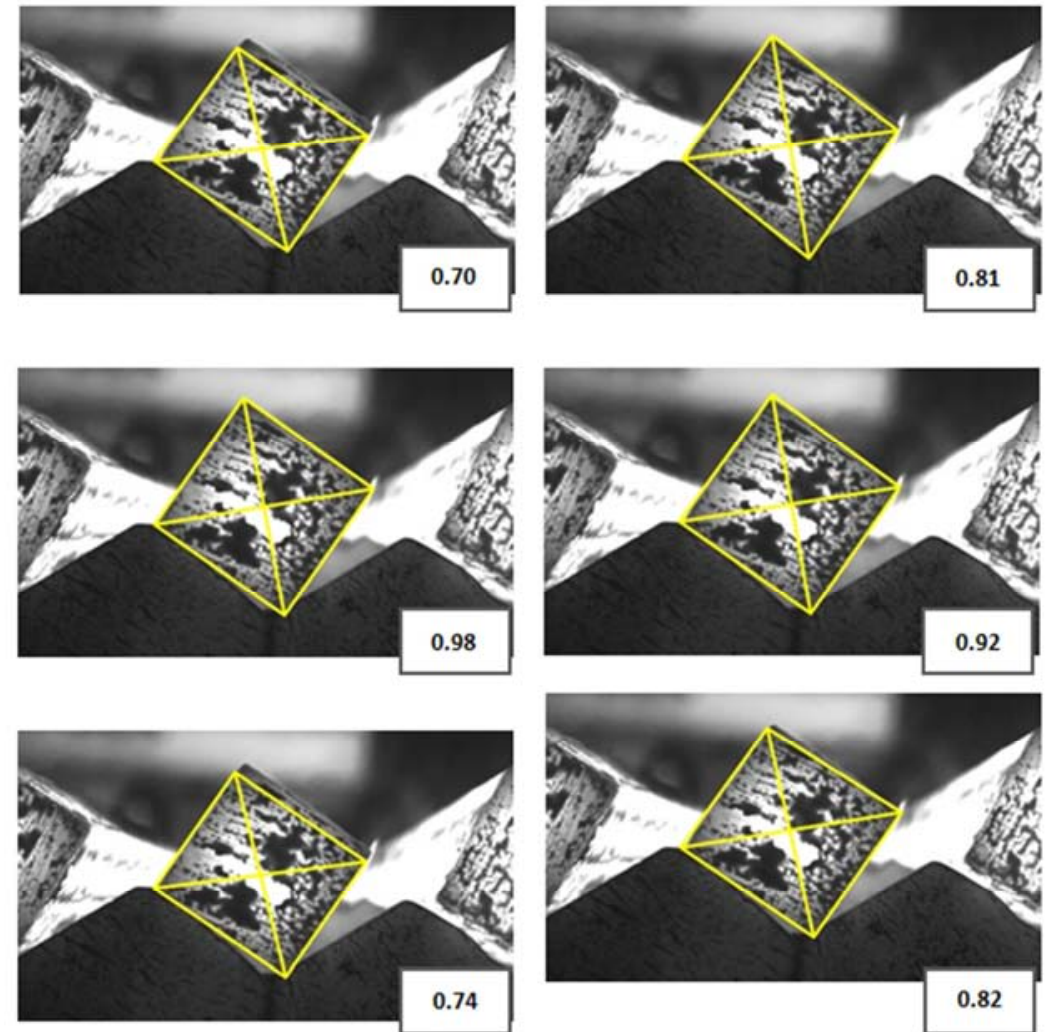

intersection of all 4 lines.

\section{$9 \times 9$ Identity Matrix} function described below. billet face.

4. Results scoring 4-line combinations:

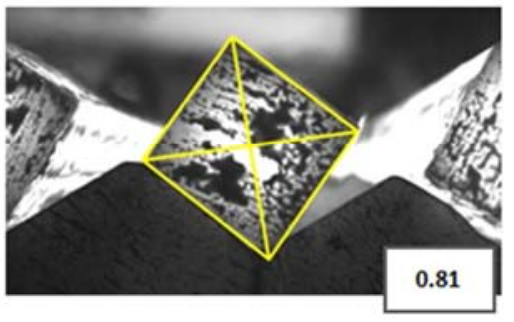

Figure 11. Results. Based on the comparison of scores the best one is chosen. In this case, one with score 0.98 has been selected as the best fit candidate.

A multivariate Gaussian penalty function with following parameters is used to provide scores to all 4-line combination:

$$
\left\{\begin{array}{c}
\mu=(130,90,130,90,130,90,130,90,16900) \\
\Sigma=\left[\begin{array}{cccc}
1 & 0 & \ldots & 0 \\
0 & 1 & \ldots & 0 \\
\vdots & \vdots & 1 & \vdots \\
0 & 0 & \ldots & 1
\end{array}\right]
\end{array}\right.
$$

The algorithm sequentially selects each 4-line combination and assigns a score to it based on the evaluation of the score

Let $\bar{x}$ be a candidate for evaluation, putting its value in the following function with known mean $(\mu)$ and covariance matrix $(\Sigma)$, the probability of that observation being generated from the Gaussian defined by Equation (8) can be used as the score for the 4-line combination to best define the

$$
P(\bar{x} \mid \mu, \Sigma)=\frac{1}{\sqrt{2 \pi|\Sigma|}} \exp \left(-\frac{1}{2}[\bar{x}-\mu]^{T} \Sigma^{-1}[\bar{x}-\mu]\right.
$$

The scores thus obtained are normalized between $(0,1)$.

The algorithm performs the exhaustive search to find the optimum solution described 4-line combination for every element in set $\mathbb{V}$. Figure 11 below shows few examples of

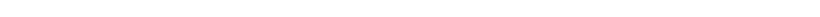

in this case, 
For $130 \mathrm{~mm}$ billet size, the mean vector $\mu$ defines the shape of an ideal square. The Covariance matrix, $\Sigma$, although, for instance, describes a unit-variance, symmetrical Gaussian and penalizes deviation from $\mu$ equally for each direction, other implementations can use lesser $\theta$ variance. This will introduce less tolerance for variations in $\theta$ as compared to the face lengths values, $l_{i}^{\prime} s$. Similarly, strict measures can be applied for the $A$ (area) variable to ensure the enclosed area tolerance remains in acceptable limits. These are design choices for implementation.

\section{Benefits}

The system has been developed and implemented at all 3 casters of LD-1 plant, viz. CC1, CC2 and CC3, Jamshedpur Works, Tata Steel, INDIA.

Generation of rhombus shape billets, adversely affects both the producer $\&$ consumer in more than one ways. It increases the yield loss by getting tons of input material rejected by the customer and it further creates an unsafe environment due to extensive material handling requirement for manual inspection. At the customer end situation is even more worse. It lowers the productivity by creating mill stoppages. It increases the energy consumption due to reheating requirements of colder billets.

The following benefits are realized after the system implementation at Jamshedpur works, Tata Steel.

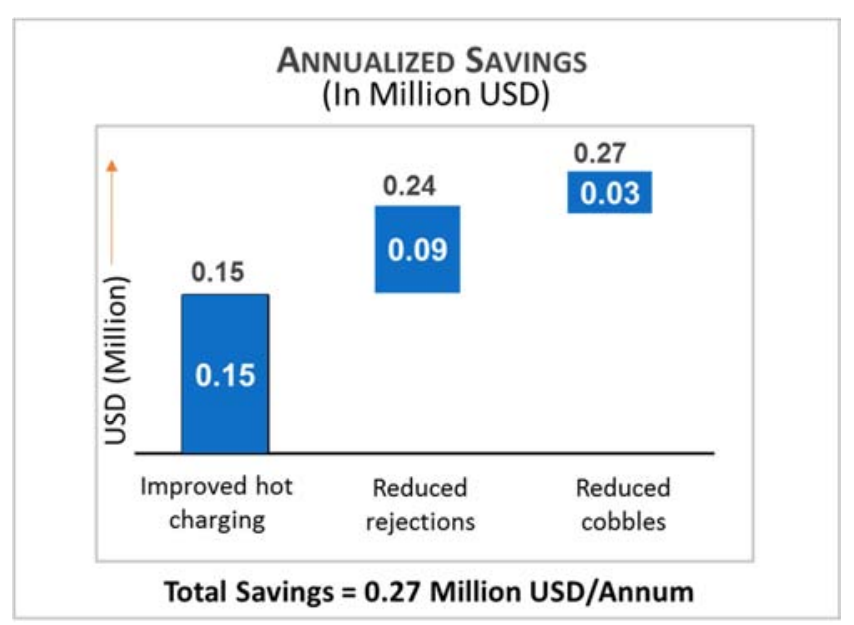

Figure 12. Annualized saving.

Under three accounts, viz. Improved hot charging, reduced rejections and reduced cobbles there is an annualized recurring saving of worth 0.27 Million USD.

Other than monetary part of it, the system has a profound influence on other aspects too. As far as efficiency is concerned, it helps reducing holding of the billets and ensures
$100 \%$ billet inspection, real-time. It saves environment by reducing rework and recycling and by removing manual inspection needs, it mitigates the safety hazards.

\section{Conclusion \& Way Forward}

Billet entanglement \& burrs, residual molten steel lumps resulting from improper torch cutting, still pose challenge in accurately determining the Rhombic distortion index for some of the billets. Although, statistical methods like KL-divergence has been used to address such issues, however, the uses of stereoscopic imaging system can be investigated further to determine billet rhomboidity.

\section{References}

[1] V. Samarasekera \& J. K. Brimacombe, The influence of mold behavior on the production of continuously cast steel billets. Metallurgical Transactions B, March 1982, Volume 13, Issue 1, pp 105-116.

[2] S. Kumar, J. A. Meech, I. V. Samarasekera, J. K. Brimacombe \& V. Racosevic, Development of intelligent mould for online detection of defects in steel billets. Ironmaking \& Steelmaking - Process, Product \& Application, Volume-26, pp 269-284.

[3] Kegham M. Markarian \& Robert Sobolewski (1981), Distortion measurement in casting, US Patent US4538669A.

[4] Markus Schmid \& Adolf Fuchs (1975). Apparatus for measuring the geometry of the hollow mold compartment of continuous casting molds, US Patent US4087918A.

[5] P. V. C. Hough. Method and means for recognizing complex patterns, US Patent 3069654.

[6] Richard O. Duda and Peter E. Hart. Use of the hough transformation to detect lines and curves in pictures. Commun. ACM, 15 (1): 11-15, January 1972.

[7] D. H. Ballard. Generalizing the hough transform to detect arbitrary shapes. pages 714-725, 1987.

[8] John Canny. A computational approach to edge detection. Pattern Analysis and Machine Intelligence, IEEE Transactions on, PAMI-8 (6): 679-698, Nov. 1986.

[9] S. Kullback, Information theory and statistics, Dover Publications, New York, 1968.

[10] K. Weinberger, J. Blitzer, and L. Saul, Distance metric learning for large margin nearest neighbor classification, in Proc. NIPS, Y. Weiss, B. Scho"lkopf, and J. Platt, Eds. Cambridge, MA: MIT Press, 2006, pp. 1475-1482.

[11] Daniel C. Cole, Reinvestigation of the thermodynamics of blackbody radiation via classical physics, Phys. Rev. A 45, 8471 - Published 1 June 1992. 